\title{
Status of the OPERA search for muon-neutrino to tau-neutrino oscillations
}

\author{
Marilisa De Serio ${ }^{* \dagger}$ \\ University of Bari \& I.N.F.N. Bari, I-70126 Bari, Italy \\ E-mail: Marilisa.Deserio@ba.infn.it
}

Updated results of the OPERA neutrino experiment, designed to perform a unique appearance measurement in the CNGS beam to confirm the oscillation mechanism in the atmospheric sector, are presented. Runs were successfully carried out in 2008, 2009, 2010 and 2011. The status of the analysis is reported and the topology and the kinematics of a second $v_{\tau} \mathrm{CC}$ candidate event, detected in the 2010-2011 sample, are described.

36th International Conference on High Energy Physics

4-11 July 2012

Melbourne, Australia

${ }^{*}$ Speaker.

On behalf of the OPERA Collaboration. 


\section{Introduction}

Over past decades, several experiments, observing solar ([1]) and atmospheric ([2]) neutrinos, as well as using artificial sources, such as nuclear reactors ([3]) and accelerators ([4]), have contributed to establish a solid framework where the disappearance of neutrinos with respect to the expected flux at a given distance from the source is well described in terms of mixing of non degenerate mass eigenstates. As they evolve in time with different phase factors, the flavour composition of a propagating beam of given energy $\mathrm{E}$ changes with the travelled distance $\mathrm{L}$ according to an oscillation probability that can be written, in the simplest two-flavor mixing scheme, as

$$
\mathrm{P}_{v_{\alpha} v_{\beta \neq \alpha}}=\sin ^{2}(2 \theta) \sin ^{2}\left(1.27 \Delta \mathrm{m}^{2} \mathrm{~L} / \mathrm{E}\right)
$$

where $\theta$ is the mixing angle, $\Delta \mathrm{m}^{2}$ is the squared mass difference in $\mathrm{eV}^{2}$ of the mixing mass eigenstates and the experimental ratio $\mathrm{L} / \mathrm{E}$ is expressed in $(\mathrm{km} / \mathrm{GeV})$.

OPERA[5] (Oscillation Project with Emulsion-tRacking Apparatus) has been designed to provide an unambiguous proof of the oscillation mechanism in the atmospheric sector by observing the appearance of oscillated $v_{\tau}$ in an almost pure high-energy $v_{\mu}$ beam (CNGS, [6]), produced at CERN-SPS and sent to the Gran Sasso underground laboratory (LNGS, Italy) at a distance of $730 \mathrm{~km}$ from the neutrino source.

\section{The OPERA experiment}

\subsection{The CNGS beam}

The CNGS beam is a conventional wide-band $v_{\mu}$ beam with an average neutrino energy of $17 \mathrm{GeV}$, an overall contamination from $\bar{v}_{\mu}, v_{e}$ and $\bar{v}_{e}$ below $3 \%$ and a negligible $v_{\tau}$ contamination. The beam energy spectrum has been designed to maximise the number of observable $v_{\tau}$ at LNGS resulting from the convolution of the oscillation probability and the neutrino cross-sections. One CNGS cycle lasts $6 \mathrm{~s}$ and contains two fast extractions, $10 \mu \mathrm{s}$ long, separated by $50 \mathrm{~ms}$. The integrated protons on target (pot) and the SPS efficiency in the runs $2008-2011$ are reported in table 1 . The performace of the machine steadly improved with increasing accumulated intensity from 2008 to 2011 , though the nominal value of $4.5 \times 10^{19}$ pot was exceeded in 2011 only. At the time of writing, the 2012 run is ongoing. Due to more stringent constraints on radiation limits, the expected integrated intensity at the end of the run will reach $\sim 18.2 \times 10^{19}$ pot instead of almost $19 \times 10^{19}$ pot, as foreseen, corresponding to about $81 \%$ of the design value.

\subsection{The $v_{\tau}$ detection principle}

The experimental signature of oscillated $v_{\tau}$ appearance consists in the detection of the $\tau$ leptons produced in charged-current $v_{\tau}$ interactions and decaying in one prong (muon, single hadron or electron) or in three prongs. The challenge of OPERA is the separation, on an eventby-event basis, of such interactions, suppressed by the oscillation probability, from dominant $v_{\mu}$ interactions. In order to resolve the $\tau$ decay topologies, extending over typical distances of a few hundred microns, with adequate background rejection power and to compensate for the 


\begin{tabular}{|c|c|c|}
\hline Run & Protons on target & SPS efficiency \\
\hline 2008 & $1.78 \times 10^{19}$ & $61 \%$ \\
\hline 2009 & $3.52 \times 10^{19}$ & $70 \%$ \\
\hline 2010 & $4.04 \times 10^{19}$ & $81 \%$ \\
\hline 2011 & $4.84 \times 10^{19}$ & $78 \%$ \\
\hline
\end{tabular}

Table 1: CNGS performance.

small neutrino interaction cross-section with matter, a target mass of the order of kilotons and an exceptional spatial resolution in the vertex region are required. The use of nuclear emulsion films, acting as sub-micrometric tracking devices, combined with a passive high density target providing the required mass has been adopted to satisfy both requirements.

\subsection{The OPERA detector}

The OPERA detector [7], shown in figure 1, is made of two identical super-modules, each one consisting of a target section followed by a muon spectrometer.

The target, with a total mass of $1.25 \mathrm{kt}$, has a modular structure and is segmented into 150000 basic units called bricks. A brick consists of 57 thin nuclear emulsion films interleaved with 56 $1 \mathrm{~mm}$-thick lead plates with transverse sizes of $12.8 \times 10.2 \mathrm{~cm}^{2}$ and dimension along the beam direction of $7.9 \mathrm{~cm}$, corresponding to about 10 radiation lengths. Bricks are arranged in 31 planar structures per super-module, called walls, with transverse dimensions of $\sim 6.7 \times 6.7 \mathrm{~m}^{2}$. Each wall, hosting $64 \times 52$ bricks, is coupled to a pair of tracker planes (TT), providing bi-dimensional track information, made of plastic scintillator strips of $2.6 \mathrm{~cm}$ width. TT planes have the primary tasks of providing the event trigger signal and identifying in real-time the brick where the $v$ interaction has occurred. A doublet of special emulsion films, called Changeable Sheets (CS, [8]), glued on the downstream face of each brick, acts as an interface between the electronic tracker with a spatial accuracy of $1 \mathrm{~cm}$ and the emulsion/lead target with sub-micrometric resolution: if tracks compatible with the TT hits are found in the analysis of the CS, the brick is disassembled and the emulsion films are developed and sent to the scanning laboratories in Europe and in Japan, equipped with high-speed automated microscopes ([9],[10]), for event location and decay search analysis [11].

The spectrometer of each super-module is a dipolar magnet $\left(\sim 8.75 \times 8 \mathrm{~m}^{2}\right)$ made of two magnetized iron walls producing a field of $1.53 \mathrm{~T}$ with vertical lines of opposite directions in the two walls. The magnet is instrumented with planes of bakelite RPC's, inserted between the iron slabs of each wall to measure the range of stopping particles and track penetrating muons. The muon charge and momentum are measured by planes of drift tubes with a spatial resolution of $\sim 300 \mu \mathrm{m}$, placed in front, behind and in between the magnet walls .

In the relevant momentum range, the resulting charge misidentifaction probability $(\sim 0.3 \%)$ is adequate for an efficient rejection of the background due to the production of charmed particles in $v_{\mu}$ interactions. The muon momentum resolution is better than $20 \%$. 


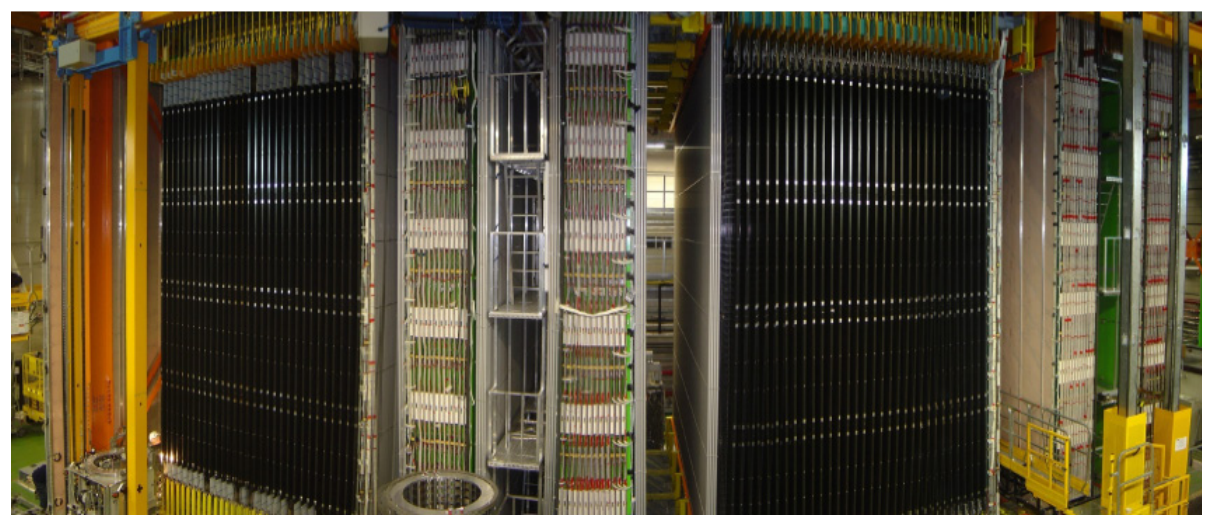

Figure 1: The OPERA detector installed in the Hall C of LNGS.

The muon identification efficiency, obtained by a combined analysis of spectrometer and scintillator tracker data, is $\sim 95 \%$.

\section{Status of data-taking and oscillation analysis results}

The results of the analysis of the data sample collected in 2008 and 2009 runs have been presented in detail in [12]: out of 2738 events located in the target and fully analysed to search for short-lived particle decays, one event compatible with the hyphothesis of being a $v_{t}$-induced CC interaction with subsequent decay of the $\tau$ lepton in one charged hadron has been detected [13], representing the first direct confirmation of the oscillation mechanism in appearance mode. The decay is consistent with the hyphothesis $\tau \rightarrow \rho v_{\tau}$. The probability that the event be due to a statistical fluctuation of the background has been evaluated to be of $5 \%$ if only the single-prong hadron channel is taken into account. Considering all decay channels, the probability for the event to be a background fluctuation is of $15 \%$.

The analysis of 2010 and 2011 data is currently in progress. While an inclusive analysis has been carried out for the runs 2008 and 2009 with all predicted events studied in order to assess the efficiencies, the following strategy is now being applied:

- all NC-like events are analysed, as well as CC-like events with a reconstructed negative muon with momentum below $15 \mathrm{GeV} / \mathrm{c}$;

- for each event, only the first predicted brick, namely the brick with the highest probability of containing the neutrino interaction point, is measured.

The first condition defines a $v_{\tau}$-enriched sample with a negligible signal loss and a significant reduction $(\sim 25 \%)$ of the $v_{\mu}$-induced interaction sample. Restricting the analysis to the first predicted bricks further reduces the emulsion scanning load.

Up to now, the study of 1343 events satisfying the criteria reported above has been completed. In total, 4126 events have been fully analysed.

In this sample, $49 v_{\mu} \mathrm{CC}$ interactions with a charmed particle in the final state have been detected, consistently with the MC expectation of $51 \pm 7$ events. Figure 2 shows the 

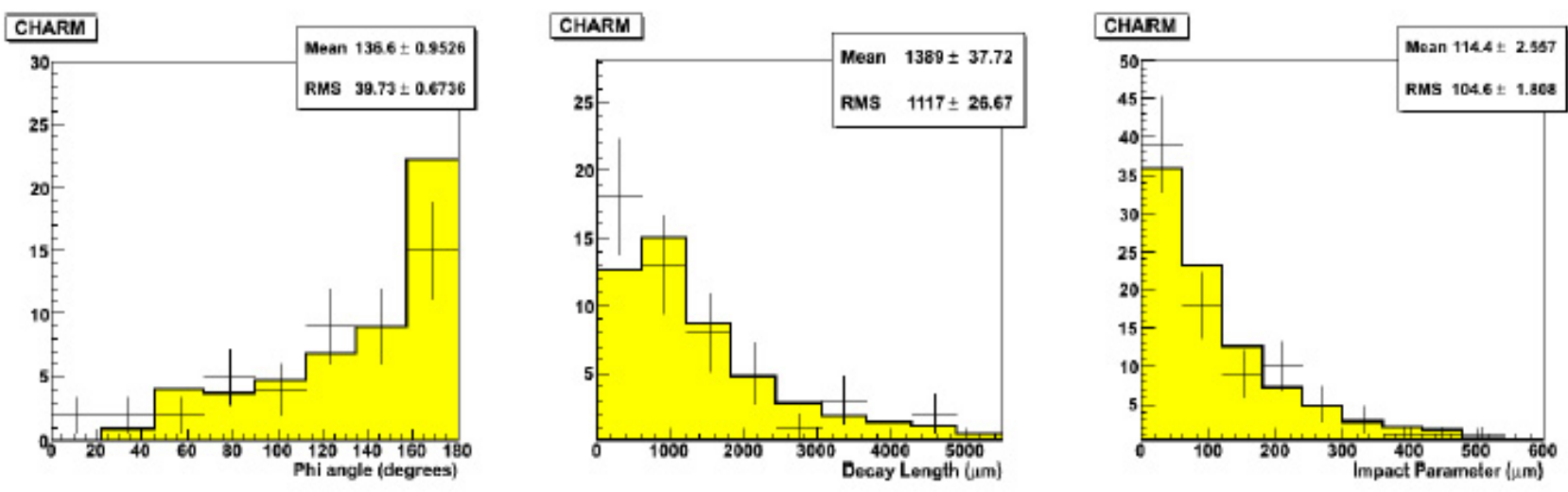

Figure 2: Comparison between observed $v_{\mu}$ interactions with charm production and decay and MC expectations. Left: distributions of the angle between the charmed particle and the primary muon in the $v$ transverse plane. Middle: distributions of the charmed particle decay length. Rigth: distributions of the charmed particle impact parameter with respect to the neutrino interaction point. Dots with error bars refer to experimental data, simulated data are represented by histograms.

comparison between data (dots with error bars) and MC (histogram) for the observed 49 events: the distributions of the angle between the charmed hadron and the muon in the plane trasverse to the neutrino direction are shown in the left plot; the distributions of the decay length and impact parameter of the charmed particle with respect to the $v$ interaction point are shown in the middle and right plots, respectively. The agreement between experimental and simulated data is a key point for OPERA, since charmed particles have lifetimes similar to that of the $\tau$ lepton showing analogous decay topologies. The decay finding efficiency is therefore similar for both types of particles and the charm sample is thus a powerful tool to monitor the $v_{\tau}$ detection capability.

In the 2010-2011 sample analysed so far, a second $v_{\tau}$ CC candidate event has been detected. A 2-prong $v$ vertex was located in lead, with an additional highly ionising nuclear fragment produced in the interaction. The two minimum ionising particles form an angle of $168^{\circ}$ in the neutrino transverse plane and one of them shows a 3-prong vertex in the plastic base of a downstream emulsion film, $1.5 \mathrm{~mm}$ after the primary $v$ interaction. One of the secondary tracks is a hadron interacting $1.3 \mathrm{~cm}$ downstream of the decay point; the other two daughter tracks, as well as the other primary track, have been identified as being hadrons based on the consistency between the range and the momentum measured in emulsion with the method described in [14]. The event satisfies all selection criteria required for $v_{\tau}$ CC interactions with $\tau \rightarrow h^{+} h^{-} h^{-} v_{\tau}$, as shown in table 2 , where the values of the relevant kinematical variables and the corresponding cuts applied for the specific decay channel are reported.

Detailed results on $v_{\mu} \rightarrow v_{\tau}$ oscillation analysis will be published in a forthcoming paper after the completion of the sample currently under study.

\section{Conclusions}

OPERA is successfully collecting data since 2008. At the end of the ongoing 2012 run, an 


\begin{tabular}{|c|c|c|}
\hline Variable & Cut & Value \\
\hline Phi Tau - Hadron (degree) & $>90$ & $167.8 \pm 1.1$ \\
\hline Average kink angle $(\mathrm{mrad})$ & $<500$ & $87.4 \pm 1.5$ \\
\hline Total momentum at decay vertex $(\mathrm{GeV} / \mathrm{c})$ & $>3.0$ & $8.4 \pm 1.7$ \\
\hline Minimum invariant mass $\left(\mathrm{GeV} / \mathrm{c}^{2}\right)$ & $>0.5,<2.0$ & $0.96 \pm 0.13$ \\
\hline Invariant mass $\left(\mathrm{GeV} / \mathrm{c}^{2}\right)$ & $>0.5,<2.0$ & $0.80 \pm 0.12$ \\
\hline Transverse momentum at $v$ vertex $(\mathrm{GeV} / \mathrm{c})$ & $<1.0$ & $0.31 \pm 0.11$ \\
\hline
\end{tabular}

Table 2: Relevant kinematical variables of the second $v_{\tau}$ candidate event detected in the 2010-2011 sample.

integrated intensity of $\sim 18.2 \times 10^{19}$ pot will have been accumulated, corresponding to about $81 \%$ of the design value. The analysis of the inclusive 2008-2009 data sample has been completed. The location and decay search of a $v_{\tau}$-enriched sample of 2010-2011 events is in progress. At the time of writing, $4126 v$ interactions have been fully studied and 2 events compatible with the hypothesis of being oscillated $v_{\tau}$ CC interactions with subsequent decay of the $\tau$ lepton in one and in three hadrons have been detected.

\section{References}

[1] Q. R. Ahmad et al. (SNO), Phys. Rev. Lett. 89 (2002) 011301.

[2] Y. Fukuda et al. (Super-Kamiokande), Phys. Rev. Lett. 81 (1998) 1562; J. Hosaka et al. (Super-Kamiokande), Phys. Rev. D 74 (2006) 032002.

[3] M. Apollonio et al. (CHOOZ), Phys. Lett. B, 466 (1999) 415; S. Abe, et al. (KamLAND), Phys. Rev. Lett. 100 (2008) 221803.

[4] M. H. Ahn, et al. (K2K), Phys. Rev. D 74 (2006) 072003; P. Adamson et al. (MINOS), Phys. Rev. Lett. 108 (2012) 191801.

[5] M. Guler et al. (OPERA), CERN-SPSC-2000-028.

[6] CNGS project, http://proj-cngs.web.cern.ch/proj-cngs.

[7] R. Acquafredda et al. (OPERA), JINST 3 (2009) P04018.

[8] A. Anokhina et al. (OPERA), JINST 3 (2008) P07005.

[9] K. Morishima, T. Nakano, JINST 5 (2010) P04011.

[10] N. Armenise et al., Nucl. Instr. Meth. A 551 (2005) 261; L. Arrabito et al., Nucl. Instr. Meth. A 568 (2006) 578.

[11] N. Agafonova et al. (OPERA), JINST 4 (2009) P06020;

A. Ariga et al., OPERA note 128, http://operaweb.Ings.infn.it:2080/Opera/publicnotes/note128.pdf.

[12] N. Agafonova et al. (OPERA), New J. Phys. 4 (2012) 033017.

[13] N. Agafonova et al. (OPERA), Phys. Lett. B 691 (2010) 138.

[14] N. Agafonova et al. (OPERA), New J. Phys. 14 (2012) 013026. 